\title{
Factors influencing utilization of cervical cancer screening services among women - A cross sectional survey
}

\author{
Jisa George T \\ College of Nursing, All India Institute of Medical Sciences (AIIMS), Bhopal, Madhya Pradesh, 462020, India
}

\section{A R T I C L E I N F O}

\section{Keywords:}

Cervical cancer

Utilization

Screening

Women

Rural

\begin{abstract}
A B S T R A C T
Introduction: Globally cervical cancer is the second most common cancer among women. Prevention and control of cervical cancer is a major public health concern. Pap smear screening is a proven and cost-effective preventive strategy for early detection of cervical cancer. Current study aimed to assess various factors affecting utilization of cervical cancer screening services.

Materials and methods: This cross-sectional study involved random selection of 430 women in the age group of 30-60 years of age residing in selected tribal settlements of Marayoor Panchayat of Idukki district of Kerala, India. Structured questionnaire was used to collect data on demographic variables, and factors affecting utilization of cervical cancer screening services.

Results: Utilization of cervical cancer screening services was very low in the studied population and only $5 \%$ of the studied population has undergone Pap smear screening during their life time. In the current study lower level of knowledge regarding cervical cancer screening was significantly associated with nonparticipation in screening services. Lack of awareness (25.06\%), lack of adequate health care facilities (22.67\%), lack of symptoms (11.69\%), not feeling at risk (11.93\%) and social stigma $(9.55 \%)$ were identified as important factors affecting utilization of cervical cancer screening services.

Conclusion: Despite being preventable low awareness level on cervical cancer and underutilization of screening services was found in the studied population and various sociodemographic and cultural factors are affecting utilization of screening services. There is a need to strengthen existing screening programmes and to implement effective campaigns to improve access to screening services.
\end{abstract}

\section{Introduction}

Cervical cancer is the leading cause of death worldwide, particularly in developing countries like India. More than $80 \%$ of the global burden of the disease is occurring in developing and underdeveloped countries due to ineffective control measures. ${ }^{1,2}$ But high income countries have achieved impressive screening coverage and reduction in cervical cancer incidence and mortality by effectively incorporating Pap smear based screening services to medical and health services. But the scenario is different in developing and underdeveloped countries, where only small percentage of the eligible population undergoes regular screening periodically. ${ }^{3}$ Moreover, health care infrastructure in low income countries can't afford the cost of implementing regular screening because of nonavailability of trained manpower and lack of resources. ${ }^{4}$ In fact, both primary and secondary preventive strategies are highly effective in preventing cervical cancer. Screening and early detection is very effective in preventing cervical cancer cases among women. Early diagnosis and treatment of precancerous lesions are associated with better cure rate and prognosis, whereas failure to detect precancerous lesions increases premature death, morbidity and mortality associated with the disease. Regular Pap smear screening can identify precancerous changes which can be cured before they turn into cancer. ${ }^{5}$ Prevention of cervical cancer by screening is a central public health concern as so many cervical related deaths are occurring each year.

It is estimated that cervical cancer burden is higher among women from low socioeconomic status and rural women in India. Lack of awareness and inaccessibility to adequate screening services are reported as the primary reason for enormous burden of the disease among rural Indian women. ${ }^{6,7}$ So many sociocultural barriers are also preventing cervical cancer screening in developing countries. Cultural factors associated with nonparticipation in cervical cancer screening are embarrassment, fear of test result, lack of support from husbands and family, inappropriate attention given to women's health issues and lack of female screening providers. ${ }^{8,9,10,11}$ Moreover, social stigma has been

E-mail address: jisagt@gmail.com. 
identified as a major barrier of cervical screening because of the belief that cervical cancer is linked with multiparity, multiple sexual partners, poor hygiene and use of oral contraceptives. A hospital based pilot study conducted in central India by recruiting 812 women attending Obstetrics and Gynaecology OPD identified insufficient public health education, sociocultural customs and beliefs, lack of patient friendly screening services, and personal factors like economic issues and lack of support from husbands and family as major factors increasing nonattendance in cervical cancer screening services. ${ }^{7}$ Based on available literature no studies are available on factors influencing cervical cancer screening behaviour among women from Kerala, India where cervical cancer is the leading cause of mortality among women. ${ }^{12}$ Therefore, present study was undertaken to assess various factors affecting utilization of cervical cancer screening services in a selected rural community of Kerala, India. Need based intervention programmes and other policies has to be developed to deal with factors leading to underutilization of cervical cancer screening services and improve rate of screening among eligible women.

\section{Materials and methods}

A descriptive cross-sectional study has been conducted to assess various factors influencing cervical cancer screening behaviour among women. 430 married women in the age group of 30-60 years of age was selected as study subjects. Study was carried out in a selected rural community of Marayoor Pachayath of idukki district, Kerala, India. Multistage cluster sampling was used to select study subjects from 6 tribal settlements of Marayoor Panchayath of Idukki district, Kerala, India. Women in the age group of 30-60 years of age, able to understand Malayalam and who are willing to participate in the study were selected as subjects.

Sociodemographic datasheet was used to collect demographic characteristics of study subjects. Structured questionnaire was used to collect data regarding various factors affecting utilization of cervical cancer screening services. Appropriate validity and reliability of data collection tools were established prior to data collection. Tool was first prepared in English and then translated to Malayalam. Language consistency was established by translation-retranslation method. Pilot study was conducted prior to final study to assess feasibility. Ethical clearance for conducting the study was taken from institutional ethics committee. Permission to carry out research study was taken from Directorate of Health services, Kerala state and District Medical officer, Idukki district Kerala. Data collection period extended from August 2016 to March 2017. Written informed consent was taken from each subject prior to data collection. Collected data was analysed using appropriate descriptive and inferential statistics using SPSS version 21. Tests of significance were performed at 0.05 level of significance and $95 \%$ confidence interval.

\section{Results}

More than half of the subjects, 246 (57.2\%) were in the age group of $30-40$ years. Lowest proportion of subjects (13.5\%) belonged to the age group of 50-60 years of age. With regard to religion majority 383 (89.1\%) were Hindus. Most of the subjects 319 (74.2\%) were from nuclear family.

More than two third of the subjects, 273 (63.5\%) were between 16 20 years and $17.8 \%$ of subjects were in the age group of $12-15$ years at the time of marriage. Only $5.8 \%$ of subjects were above the age of 26 years at the time of marriage. With regard to age at the time of first pregnancy, $23.3 \%$ of subjects were below 18 years at the time of first pregnancy. More than half of subjects (55.3\%) were between the age group of 18-21 years at the time of first pregnancy. $1.4 \%$ of subjects were nulliparous. More than half of subjects (51.9\%) had monthly income between 1500 - 4500 , followed by $37.2 \%$ had monthly income between $4500-7600$ and only $1.2 \%$ had between $11300-15200$. Majority of subjects were ( $44.7 \%$ \& $42.8 \%)$ either housewives or labourers.
Table 1

Level of awareness regarding cervical cancer and screening services. $N=430$.

\begin{tabular}{lll}
\hline Variables & Frequency & Percentage \\
\hline Level of awareness of cervical cancer & & \\
Good & 5 & 1.16 \\
Average & 40 & 9.30 \\
Poor & 385 & 89.54 \\
Level of awareness of cervical cancer screening & & \\
Good & 0 & 0 \\
Average & 46 & 10.70 \\
Poor & 384 & 89.30 \\
\hline
\end{tabular}

Utilization of cervical cancer screening services was very low in the studied population. Study findings revealed that only $5 \%$ of the studied population has undergone Pap smear screening during their life time. In the present study lower level of knowledge regarding cervical cancer screening was significantly associated with screening behaviour of women. Table 1 explains level of awareness of women regarding cervical cancer and screening services.

Present study also assessed various factors affecting utilization of cervical cancer careening services among women. Fig. 1 explains various factors affecting utilization of cervical cancer screening services among women. Lack of awareness and lack of adequate health care facilities were identified as major factors $(25.06 \%$ \& $22.67 \%$ ) affecting utilization of Pap smear screening services. Equal proportion of subjects $(11.93 \% \& 11.69 \%)$ reported lack of symptoms and not feeling at risk as a factor contributing to lack of screening behaviour. Least number of subjects (1.43\%) reported fear of vaginal examinations as a factor influencing nonutilization of Pap smear screening.

In the current study significant association was found between cervical cancer screening behaviour with age at the time of marriage $(p=0.003)$ and age at the time of pregnancy $(p=0.004)$. Higher age at the time of marriage and first pregnancy was associated with good screening behaviour. Educated and women belonging to higher socioeconomic status showed good compliance with cervical cancer screening ( $\mathrm{p}=0.000$ and $\mathrm{p}=0.002$ ). Higher number of parity, long duration of marriage and age at the time of marriage was also significantly associated with cervical cancer screening $(\mathrm{p}=0.025, \mathrm{p}=$ $0.019, \mathrm{p}=0.003$ ) Table 2 shows association between cervical cancer screening behaviour of the women with selected sociodemographic variables.

Table 2

Association between cervical cancer screening behaviour of women with selected sociodemographic variables. $\mathrm{N}=430$.

\begin{tabular}{llll}
\hline SI. No & Variable & Chi- Square Value & p-value \\
\hline 1. & Age & 1.154 & $0.573^{\text {ns }}$ \\
2. & Education & 30.700 & $0.000^{\mathrm{c}}$ \\
3. & Income & 14.653 & $0.002^{\mathrm{b}}$ \\
4. & Occupation & 8.659 & $0.055^{\mathrm{ns}}$ \\
5. & Age at the time of marriage & 13.412 & $0.003^{\mathrm{b}}$ \\
6. & Age at the time of first pregnancy & 14.9111 & $0.004^{\mathrm{b}}$ \\
7. & Number of pregnancies & 8.972 & $0.025^{\mathrm{a}}$ \\
8. & Number of children & 14.047 & $0.002^{\mathrm{b}}$ \\
9. & Duration of marriage in years & 9.841 & $0.019^{\mathrm{a}}$ \\
\hline
\end{tabular}

Fisher's Exact Test/Chi-square test.

${ }^{n s}$ Not significant.

a Significant at 0.05 level.

b Significant at 0.01 level.

c Significant at 0.001 level. 


\section{Factors affecting Pap Smear screening}

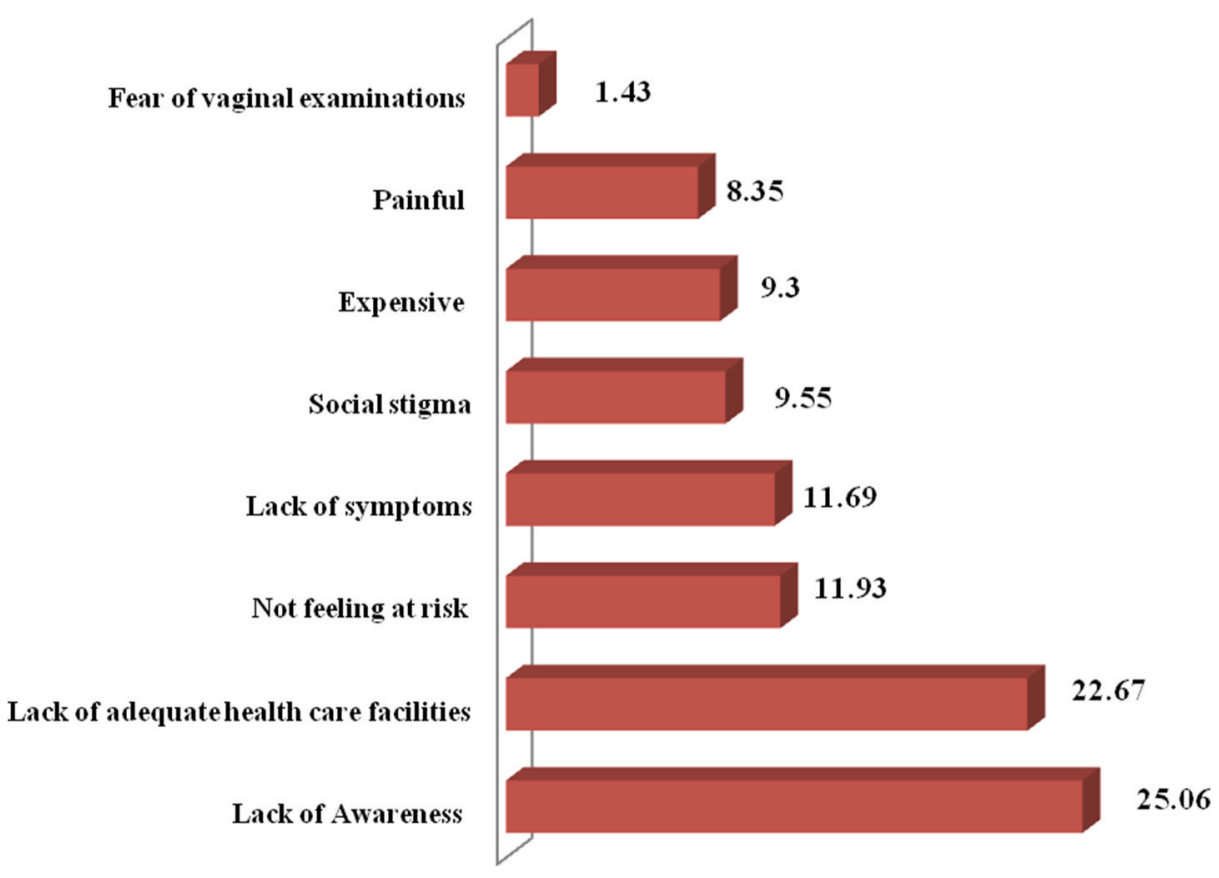

Fig. 1. Varous factors affecting utilization of cervical cancer screening services.

\section{Discussion}

Present study assessed various factors affecting utilization of cervical cancer screening services in a rural community of Idukki district, Kerala, India. Despite being preventable by appropriate screening strategies, significant underutilization of cervical cancer screening services was reported in the study. Utilization of cervical cancer screening services depends on various factors like health care associated factors, availability of trained professionals and women themselves. Current findings revealed that only $5 \%$ of the study subjects have undergone Pap smear screening during their lifetime. Concurrent findings were found in a study done among women visiting tertiary care hospital in Delhi, India in 2014 where, only $7.3 \%$ of the women interviewed had undergone pap smear screening within 3 years. ${ }^{13}$ However, another study to find out factors influencing utilization of cervical cancer screening services among married women aged 30-60 of Bharatpur, Nepal showed that $56 \%$ of women never utilized cervical cancer screening services. ${ }^{14}$

Even though cervical cancer is the second common cancer among women in India, low knowledge level was reported among participants regarding cervical cancer and Pap smear screening. Current study revealed positive relationship between knowledge regarding cervical cancer screening and utilization of screening services. So specific knowledge on cervical cancer is a critical factor influencing cervical cancer screening services and adoption of healthy lifestyle practices. This indicates the importance of support system from healthcare professionals to make patients aware of Pap smear test. In addition, awareness programmes and screening campaigns should also be arranged for rural women who have less contact with health care facilities to improve their knowledge and practices on prevention of cervical cancer. Consistent findings were reported in studies conducted in rural communities of Tamil Nadu and Kerala, India and findings revealed that low awareness level on prevention of cervical cancer was associated with non-utilization of preventive services. ${ }^{15,16,17}$ Study carried out by Roy B, and Tricia ST to assess beliefs and predictors of cervical cancer screening in Kolkata also found higher level of preventive practices on cervical cancer screening among women having good awareness on screening services. ${ }^{18}$ Kahesa et al in their study in Tanzania found that awareness about cervical cancer and existing screening programmes was associated with improved acceptance rates. ${ }^{19}$

This study identified high intake of cervical cancer screening among educated and employed women. Singh M, Ranjan R, Das B, and Gupta K in 2014 reported good practices regarding cervical cancer screening among educated and working women ${ }^{20}$ Another study which supports the findings of the present study was conducted in AIIMS, Bhopal, where women with education above secondary were more likely to execute positive practices of screening for cervical cancer. ${ }^{21}$ This is on account of more access to information about the test through colleagues and media and more opportunities to get tested. In addition, women who are employed have a greater chance for social interaction as a result they get to know more about the disease and screening services. On the other hand, contrast findings were found in a descriptive cross-sectional study conducted in a rural community of Kerala, where no significant association was seen between educational status of the women and utilization of cervical cancer screening practices. ${ }^{17}$

In the current study no significant association was found between cervical cancer screening and age of the participants. However, a study conducted in Kerala, India found increased rate of participation in cervical cancer screening programmes with increasing age. ${ }^{17}$ On the other hand, contradictory findings of increased participation in cervical cancer screening programmes among younger women was seen in studies conducted in Maharashtra, India ${ }^{22}$ and South India. ${ }^{23}$

About $25 \%$ of the subjects participated in the study never undergone Pap smear screening because of lack awareness regarding screening services. This was supported by various other studies. 7,24,25,26,27 Moreover, cervical cancer screening services are mostly available in tertiary health institutions in India which is also a factor influencing screening behaviour. Nearly $26 \%$ of the respondents not participated in regular screening programmes due to lack of adequate health care facilities in the nearby hospitals which was consistent with findings of a study carried out in India., ${ }^{7,28}$ Availability of Pap smear screening facilities in the nearby areas will increase screening uptake among rural women. ${ }^{29,30}$ 
Various socio demographic factors such as age, marital status, educational attainment, and socioeconomic status are significantly correlated with utilization of screening services. Financial constraints also restrict rural women from utilizing screening services available in tertiary care institutions. Results of the present study clearly indicated that women who were less educated and from lower socioeconomic status were least likely to be involved in screening. Consistent findings were found in a study done by ACCP partners in South Africa, Peru, Kenya, and India to investigate the determinants of screening participation. ${ }^{31}$ Studies of cervical cancer screening participation in India have recommended that women living in rural areas ${ }^{22,23}$ who are older, unmarried, and with lower educational attainment are less likely to have involved in cervical cancer screening as compared to women living in urban settings who are younger and more educated. ${ }^{22,27,32}$

In the current study social stigma has been identified as a major factor contributing to nonattendance in cervical cancer screening. Attitude regarding personal susceptibility was also identified as a factor affecting uptake of screening services. Many women in the study were reluctant to attend screening as they perceived themselves at low risk for developing disease and also as they were not having symptoms of the disease. Concurrent findings of higher rate of screening among women who perceived cervical cancer as serious disease was reported in several other studies. ${ }^{33,34,35}$ Factors such as embarrassment, fear of pelvic examination and misconceptions regarding Pap smear screening have been also identified as leading factors of not attending accessible screening services frequently. $8 \%$ of the women not utilized screening facilities as Pap smear screening is painful. ${ }^{36}$

Study found significant association between cervical cancer screening behaviour and parity of women. Findings of the study revealed high rate of compliance with cervical cancer screening among women with higher number of pregnancies indicating that frequent contact with health care facility increases awareness on screening facilities among women which was supported by findings of a studies conducted in India. ${ }^{7,23}$ However, contrary findings of less compliance with cervical cancer screening with higher number of pregnancies was reported in a study conducted in Maharashtra, India. ${ }^{22}$

So many socio demographic and cultural factors are influencing cervical cancer screening uptake among women in developing countries like India. Therefore, it very essential to use appropriate service delivery mechanisms to ensure good participation of women in screening services especially in rural areas of India. It is extremely important to consider screening as an important component when making public health policies and implementation of cervical cancer control programs. Women who have never been screened need basic education about cancer screening and increasing access to care. Effectively organised, population-based screening programs need to be established in developing countries to improve screening behaviour and to reduce burden associated with cervical cancer. In the current scenario, Human Papilloma Virus (HPV) vaccine is a recommended strategy to prevent cervical cancer. Future studies can be conducted to evaluate the awareness and role of HPV vaccine in cervical cancer prevention.

\section{Conclusion}

Study revealed limited knowledge of Indian women about cervical cancer prevention and screening. Sociocultural practices and beliefs, lack of adequate screening facilities and infrastructure for providing screening services, lack of adequately trained manpower, and personal difficulties in accessing appropriate health care facilities were identified as foremost barriers influencing screening behaviour among eligible women. There is an utmost priority for different organizational and educational policies and approaches to be used to improve the reproductive health of women. Therefore, it is essential to device and implement need based and culturally specific community education and screening programmes by involving community leaders to minimize sociocultural factors affecting cervical cancer screening and to improve compliance with cervical cancer screening services among rural women.

\section{Conflict of any interest}

Nil.

\section{Ethical approval and consent to participate}

Ethical Clearance for conducting the study was taken from Institutional ethics committee and written informed consent was taken from the participants prior to data collection.

\section{Author contribution}

Significant contribution to the concept, design, literature search, data acquisition, data analysis and manuscript preparation.

\section{References}

1 Bruni L, BarrionuevoRosas L, Albero G, Aldea M, Serrano B, Valencia S. ICO information centre on HPV and cancer (HPV information centre). In: Human Papillomavirus and Related Diseases in the World. Summary Report. July 2017, 27.

2 World Health Organization. WHO/ICO Information Centre on HPV and Cervical Cancer (HPV Information Centre): Summary Report 2010. Geneva: World Health Organization; 2010.

3 Sahasrabuddhe VV, Parham GP, Mwanahamuntu MH, Vermund SH. Cervical cancer prevention in low-and middle-income countries: feasible, affordable, essential. Canc Prev Res. 2012 Jan;5(1):11-17, 1.

4 Julinawati S, Cawley D, Domegan C, Brenner M, Rowan NJ. A review of the perceived barriers within the health belief model on pap smear screening as a cervical cancer prevention measure. J Asian Sci Res. 2013 Jun;3(6):677, 1.

5 Demarteau N, Morhason-Bello IO, Akinwunmi B, Adewole IF. Modeling optimal cervical cancer prevention strategies in Nigeria, 24 BMC Canc. 2014 May;14(1):365. Available from https://bmccancer.biomedcentral.com/articles/10.1186/1471-240714-365.

6 Arbyn M, Castellsagué X, de Sanjosé S, et al. Worldwide burden of cervical cancer in 2008, 6 Ann Oncol. 2011 Apr;22(12):2675-2686. Available from: URL https://acade mic.oup.com/annonc/article/22/12/2675/212678/Worldwideburden-of-cervical-ca ncer-in-2008.

7 Singh S, Badaya S. Factors influencing uptake of cervical cancer screening among women in India: a hospital based pilot study. J Community Med Health Educ. 2012;2 (157). https://doi.org/10.4172/2161-0711.1000157.

8 Lee SY. Cultural factors associated with breast and cervical cancer screening in Korean American women in the US: an integrative literature review. Asian Nurs Res. 2015 Jun;9(2):81-90, 30.

9 Julinawati S, Cawley D, Domegan C, Brenner M, Rowan NJ. A review of the perceived barriers within the health belief model on pap smear screening as a cervical cancer prevention measure. J Asian Sci Res. 2013 Jun;3(6):677, 1.

10 Denny L, Prendiville W. Cancer of the cervix: early detection and cost-effective solutions. Int J Gynaecol Obstet. 2015 Oct;131:S28-S32, 31.

11 American Cancer Society. Cancer Prevention \& Early Detection Facts \& Figures 20152016. Atlanta: American Cancer Society; 2015.

12 Nandakumar A, Ramnath T, Chaturvedi M. The magnitude of cancer cervix in India. Indian J Med Res. 2009 Sep;130:219-221.

13 Singh M, Ranjan R, Das B, Gupta K. Knowledge, attitude and practice of cervical cancer screening in women visiting a tertiary care hospital of Delhi. Indian J Canc. 2014;51(3):319-323.

14 Ranabhat MK, Thapa B. Factors influencing utilization of cervical cancer screening services among married women in community, Chitwan. Journal of Chitwan Medical College. 2017 Sep;7(3):42-49, 30.

15 Mani G, Annadurai K, Danasekaran R. Awareness regarding cervical cancer and preventive practices among rural married women of kancheepuram district, Tamil Nadu. J compr health. 2014;1(2):42-48.

16 Siddharthar J, Rajkumar B, Deivasigamani K. Knowledge, awareness and prevention of cervical cancer among women attending a tertiary care hospital in Puducherry. India J Clin Diagn Res. 2014 Jun;8(6):OC01-OC03.

17 Aswathy S, Quereshi MA, Kurian B, Leelamoni K. Cervical cancer screening: current knowledge \& practice among women in a rural population of Kerala, India. Indian $J$ Med Res. 2012 Aug;136(2):205-210.

18 Roy B, Tang TS. Cervical cancer screening in Kokata, India: beliefs and predictors of cervical cancer screening among women attending a women's Health Clinic in Kolkata. J Canc Educ. 2008;23:253-259.

19 Kahesa C, Mwaiselage J, Wabinga HR, et al. Association between invasive cancer of the cervix and HIV-1 infection in Tanzania: the 223 need for dual screening, 30 BMC Publ Health. 2008 Jul;8(1):262. Available from: URL https://bmcpublichealth. biomedcentral.com/articles/10.1186/1471-2458-8-262.

20 Singh M, Ranjan R, Das B, Gupta K. Knowledge, attitude and practice of cervical cancer screening in women visiting a tertiary care hospital of Delhi. Indian J Canc. 2014;51(3):319-323. 
21 Bansal AB, Pakhare AP, Kapoor N, Mehrotra R, Kokane AM. Knowledge, attitude, and practices related to cervical cancer among adult women: a hospital-based crosssectional study. J Nat Sci Biol Med. 2015 Jul-Dec;6(2):324-328.

22 Nene B, Jayant K, Arrossi S, et al. Determinants of women s participation in cervica cancer screening trial, Maharashtra, India. Bull World Health Organ. 2007 Apr;85(4): 264-272.

23 Sankaranarayanan R, Rajkumar R, Arrossi S, Theresa R, Esmy PO, etal Mahé C. Determinants of participation of women in a cervical cancer visual screening trial in rural south India. Canc Detect Prev. 2003;27(6):457-465.

24 Bessler P, Aung M, Jolly P. Factors affecting uptake of cervical cancer screening among clinic attendees in Trelawny, Jamaica. Cancer Control. 2007 Oct;14(4): 396-404.

25 Waller J, Bartoszek M, Marlow L, Wardle J. Barriers to cervical cancer screening attendance in England: a population-based survey. J Med Screen. 2009;16(4): 199-204.

26 Utoo BT, Ngwan SD, Anzaku AS. Utilization of screening services for cancer of the cervix in Makurdi, Nigeria. Reprod Health. 2013 Dec;1(1):2.

27 Kaku M, Mathew A, Rajan B. Impact of socio-economic factors in delayed reporting and late-stage presentation among patients with cervix cancer in a major cancer hospital in South India. Asian Pac J Cancer Prev APJCP. 2008 Oct;9(4):589-594.

28 Basu P, Chowdhury D. Cervical cancer screening \& HPV vaccination: a comprehensive approach to cervical cancer control. Indian J Med Res. 2009 Sep;130 (3):241, 1.
29 Thomas VN, Saleem T, Abraham R. Barriers to effective uptake of cancer screening among Black and minority ethnic groups. Int J Palliat Nurs. 2005 Nov;11(11): 562-571.

30 Burns A, Sanghvi H, Lu R, Gaffikin L, Blumenthal PD. Saving women's lives from cervical cancer. Lancet. 2011 Apr;377(9774):1318, 16.

31 Coffey P, Arrossi S, Bradley J, Dzuba I, White S. ACCP Community Involvement Affinity Group. Improving screening coverage rates of cervical cancer prevention programs: a focus on communities. In: Seattle: ACCP;Cervical Cancer Prevention Issues in Depth. vol. 4. 2004.

32 Jayaprakash M, Eralil GJ, Jose R, Radha KR, Vijilakshmi AG, Pg A. Study on knowledge and practice of screening for cervical cancer among women from rural communities in central Kerala, India. Int J Reprod Contracept Obstet Gynecol. 2018;7 (10):4165-4170.

33 Mosavel M, Simon C, Oakar C, Meyer S. Cervical cancer attitudes and beliefs- a cape town community responds on world cancer day. J Canc Educ. 2009;24(2):114-119.

34 Wong LP, Wong YL, Low WY, Khoo EM, Shuib R. Knowledge and awareness of cervical cancer and screening among Malaysian women who have never had a Pap smear: a qualitative study. Singap Med J. 2009;50(1):49-53.

35 Behbakht K, Lynch A, Teal S, Degeest K, Massad S. Social and cultural barriers to Papanicolaou test screening in an urban population. Obstet Gynecol. 2004 Dec;104 (6):1355-1361.

36 Ekane GE, Obinchemti TE, Nguefack CT, et al. Pap smear screening, the way forward for prevention of cervical cancer? A community based study in the buea health district, Cameroon. Open J Obstet Gynecol. 2015 Mar;5:226-233, 26. 